Article

\title{
Equicontinuity, Expansivity, and Shadowing for Linear Operators
}

\author{
Keonhee Lee ${ }^{1}$ and C. A. Morales ${ }^{2, *(\mathbb{D})}$ \\ 1 Department of Mathematics, Chungnam National University, Daejeon 305-764, Korea; khlee@cnu.ac.kr \\ 2 Nstituto de Matemática Universidade Federal do Rio de Janeiro, P. O. Box 68530, \\ Rio de Janeiro 21945-970, Brazil \\ * Correspondence: morales@impa.br; Tel.: +82-10-9725-7715
}

Received: 22 September 2018; Accepted: 11 November 2018 ; Published: 15 November 2018

\begin{abstract}
We prove that a linear operator of a complex Banach space has a shadowable point if and only if it has the shadowing property. In addition, every equicontinuous linear operator does not have the shadowing property and its spectrum is contained in the unit circle. Finally, we prove that if a linear operator is expansive and has the shadowing property, then the origin is the only nonwandering point.
\end{abstract}

Keywords: linear operator; banach space; shadowable point; equicontinuous

\section{Introduction}

The dynamics of linear operators on Banach spaces has been studied in the literature [1]. For instance, Eisenberg and Hedlund [2,3] characterized uniformly expansive operators in terms of spectral theory, and Ombach [4] as well as Bernardes et al. [5] tried to obtain a similar result for operators with the shadowing property. In this paper, we will continue the study of the dynamics of such operators. Indeed, we prove that a linear operator has a shadowable point (in the sense of Reference [6]) if and only if it has the shadowing property. Moreover, we prove that an equicontinuous linear operator has no shadowing property and its spectrum is contained in the unit circle. Finally, it is proved that every expansive linear operator with the shadowing property does not have nonzero nonwandering points. Let us state these results in a precise way.

Hereafter, $X$ will denote a Banach space (all such spaces will be complex). By a linear operator, we mean an operator bounded one-to-one onto a linear map with a bounded inverse. Given a $\delta>0$, a $\delta$-pseudo-orbit of a linear operator $T$ is a bi-infinite sequence $\left(x_{n}\right)_{n \in \mathbb{Z}}$ of $X$ such that $\left\|T\left(x_{n}\right)-x_{n+1}\right\| \leq$ $\delta$ for every $n \in \mathbb{Z}$. We say that $T$ has the shadowing property if for every $\varepsilon>0$ there is $\delta>0$ such that every $\delta$-pseudo orbit $\left(x_{n}\right)_{n \in \mathbb{Z}}$ can be $\varepsilon$-shadowed i.e., there is $x \in X$ such that $\left\|T^{n}(x)-x_{n}\right\| \leq \varepsilon$ for every $n \in \mathbb{Z}$. The following pointwise version of the shadowing property is given in Reference [6]. Some authors have recently been studying it [7-9]. We say that $x \in X$ is shadowable if for every $\varepsilon>0$ there is $\delta>0$ such that every $\delta$-pseudo orbit $\left(x_{n}\right)_{n \in \mathbb{Z}}$ through $x$ (i.e., $x_{0}=x$ ) can be $\varepsilon$-shadowed. Clearly, if $T$ has the shadowing property, then every point is shadowable. This motivates our first result.

Theorem 1. A linear operator of a Banach space has a shadowable point if and only if it has the shadowing property.

On the other hand, a linear operator $T$ is equicontinuous if for every $\varepsilon>0$ there is $\delta>0$ such that if $x, y \in X$ and $\|x-y\| \leq \delta$, then $\left\|T^{n}(x)-T^{n}(y)\right\| \leq \varepsilon$ for every $n \in \mathbb{Z}$. The spectrum of a linear operator $T$ is the set of those $\lambda \in \mathbb{C}$ such that $T-\lambda I$ is not invertible. 
Theorem 2. Every equicontinuous linear operator of a Banach space does not have the shadowing property and its spectrum is contained in the unit circle.

Finally, we recall that a linear operator of a Banach space $T \in \mathcal{L}(X)$ is expansive if for each $x \in X$ with $\|x\|=1$ there is $n \in \mathbb{Z}$ such that $\left\|T^{n}(x)\right\| \geq 2$. This definition is equivalent to the classical notion of expansivity on metric spaces [10]. It is known that an expansive linear operator with the shadowing property does exist on finite or infinite dimensional Banach spaces and that all of them are uniformly expansive, i.e., there is $n \in Z$ such that $\max \left\{\left\|T^{n}(x)\right\|,\left\|T^{-n}(x)\right\|\right\} \geq 2$ for every $x \in X$ with $\|x\|=1$. Indeed, every hyperbolic linear operator (i.e, the spectrum does not intersect the unit complex circle) is expansive with the shadowing property (e.g., Reference [5]).

On the other hand, linear operators with the shadowing property may have nonzero nonwandering points. Recall that a nonwandering point of a linear operator $T \in \mathcal{L}(X)$ is a point $x \in X$ such that for every neighborhood $U$ of $x$ there is $k \in \mathbb{N}$ satisfying $T^{k}(U) \cap U \neq \varnothing$. Every linear operator has at least one nonwandering point (e.g., the origin $x=0$ ) and for hyperbolic operators this is the only one. Now we can state our last result.

Theorem 3. If a linear operator is expansive and has the shadowing property, then the origin is the only nonwandering point.

The authors would like to thank the participants of the dynamical system seminar at the math department of the Chungnam National University at Daejeon, Korea, for helpful conversations.

\section{Proof of the Theorems}

Theorem 1 is actually contained in the following result. Hereafter, $X$ denotes a Banach space and $\mathcal{L}(X)$ denotes the set of linear operators $T: X \rightarrow X$.

Theorem 4. The following properties are equivalent for every $T \in \mathcal{L}(X)$.

1. Thas the shadowing property.

2. Thas a shadowable point.

3. 0 is a shadowable point of $T$.

Proof. Obviously Item (1) implies Item (2). To prove Item (2) implies Item (3), let $x$ be a shadowable point. First, we show that $-x$ is shadowable. Let $\varepsilon>0$ and $\delta$ be given by the shadowableness of $x$. Let $\left(x_{n}\right)_{n \in \mathbb{Z}}$ be a $\delta$-pseudo orbit through $-x$. It follows that $x_{0}=-x$ and $\left\|T\left(x_{n}\right)-x_{n+1}\right\| \leq \delta$, for every $n \in \mathbb{Z}$. Set $\hat{x}_{n}=-x_{n}$. Then, $\hat{x}_{0}=x$ and $\left\|T\left(\hat{x}_{n}\right)-\hat{x}_{n+1}\right\|=\left\|T\left(x_{n}\right)-x_{n+1}\right\| \leq \delta$ for every $n \in \mathbb{Z}$ proving that $\left(\hat{x}_{n}\right)_{n \in \mathbb{Z}}$ is a $\delta$-pseudo orbit through $x$. It follows that there is $z \in X$ such that $\left\|T^{n}(z)-\hat{x}_{n}\right\| \leq \varepsilon$ for every $n \in \mathbb{Z}$. Then,

$$
\left\|T^{n}(-z)-x_{n}\right\|=\left\|-\left(T^{n}(z)-\hat{x}_{n}\right)\right\|=\left\|T^{n}(z)-\hat{x}_{n}\right\| \leq \varepsilon, \quad \forall n \in \mathbb{Z},
$$

proving that $-x$ is shadowable. Now, let $\varepsilon>0$ and $\delta$ be given by the shadowableness of of $-x$. Let $\left(z_{n}\right)_{n \in \mathbb{Z}}$ be a $\delta$-pseudo orbit through 0 . Define $\hat{z}_{n}=z_{n}-T^{n}(x)$. It follows that $\hat{z}_{0}=z_{0}-x=-x$ and

$$
\left\|T\left(\hat{z}_{n}\right)-\hat{z}_{n+1}\right\|=\left\|T\left(z_{n}-T^{n}(x)\right)-\left(z_{n+1}-T^{n+1}(x)\right)\right\|=\left\|T\left(z_{n}\right)-z_{n+1}\right\| \leq \delta, \forall n \in \mathbb{Z} .
$$

It follows that there is $\hat{z} \in X$ such that $\left\|T^{n}(\hat{z})-\hat{z}_{n}\right\| \leq \varepsilon$ for every $n \in \mathbb{Z}$. Therefore, taking $z=\hat{z}+x$ we get

$$
\left\|T^{n}(z)-z_{n}\right\|=\left\|T^{n}(\hat{z}+x)-\left(\hat{z}_{n}+T^{n}(x)\right)\right\|=\left\|T^{n}(\hat{z})-\hat{z}\right\| \leq \varepsilon, \quad \forall n \in \mathbb{Z}
$$


proving (3).

Finally, we prove that Item (3) implies Item (1). Let $\varepsilon>0$ and $\delta$ be given by the shadowableness of 0 . Let $\left(x_{n}\right)_{n \in \mathbb{Z}}$ be a $\delta$-pseudo orbit and define $\hat{x}_{n}=x_{n}-T^{n}\left(x_{0}\right)$ for all $n \in \mathbb{Z}$. Then, $\hat{x}_{0}=x_{0}-x_{0}=0$ and

$$
\left\|T\left(\hat{x}_{n}\right)-\hat{x}_{n+1}\right\|=\left\|T\left(x_{n}-T^{n}\left(x_{0}\right)\right)-\left(x_{n+1}-T^{n}\left(x_{0}\right)\right)\right\|=\left\|T\left(x_{n}\right)-x_{n+1}\right\| \leq \delta
$$

for every $n \in \mathbb{Z}$. It follows that $\left(\hat{x}_{n}\right)_{n \in \mathbb{Z}}$ is a $\delta$-pseudo orbit through 0 . Hence, there is $y \in X$ such that $\left\|T^{n}(y)-\hat{x}_{n}\right\| \leq \varepsilon$ for every $n \in \mathbb{Z}$. It follows that $\hat{y}=y+x_{0}$ satisfies

$$
\left\|T^{n}(\hat{y})-x_{n}\right\|=\left\|T^{n}\left(y+x_{0}\right)-x_{n}\right\|=\| T^{n}(y)-\left(x_{n}-T^{n}\left(x_{0}\right)\|=\| T^{n}(y)-\hat{x}_{n} \| \leq \varepsilon\right.
$$

for every $n \in \mathbb{Z}$ proving Item (1). This completes the proof of the theorem.

To prove Theorem 2, we first observe that $T \in \mathcal{L}(X)$ is equicontinuous if and only if for every $\varepsilon>0$ there is $\delta>0$ such that $\|x\| \leq \delta$ implies $\left\|T^{n}(x)\right\| \leq \varepsilon$ for every $n \in \mathbb{Z}$. Another equivalence is as follows.

Lemma 1. The following properties are equivalent for every $T \in \mathcal{L}(X)$ :

1. Tis equicontinuous.

2. $\quad T$ is power bounded namely $\sup _{n \in \mathbb{Z}}\left\|T^{n}\right\|<\infty$ (see Reference [11]).

Proof. To prove that Item (1) implies Item (2), let $\delta>0$ be given by the equicontinuity of $T$ for $\varepsilon=1$. Suppose that $x \in X$ is unitary, i.e., $\|x\| \leq 1$. Then, $\|\delta x\| \leq \delta$ and so $\left\|T^{n}(\delta x)\right\| \leq 1$ for every $n \in \mathbb{Z}$. From this, we get $\left\|T^{n}(x)\right\| \leq \frac{1}{\delta}$ for every $n \in \mathbb{Z}$ and every unitary vector $x$ proving Item (2). Conversely, suppose Item (2) and take $0<M<\infty$ such that $\left\|T^{n}\right\| \leq M$ for every $n \in \mathbb{Z}$. Fix $\varepsilon>0$ and set $\delta=\frac{\varepsilon}{M}$. Then, if $\|x\| \leq \delta,\left\|\frac{x}{\delta}\right\| \leq 1$ so $\left\|T^{n}\left(\frac{x}{\delta}\right)\right\| \leq M$ for every $n \in \mathbb{Z}$ proving $\left\|T^{n}(x)\right\| \leq \delta M=\varepsilon$ for every $n \in \mathbb{Z}$. Therefore, $T$ is equicontinuous and Item (1) holds.

Denote by $S^{1}$ the unit circle in $\mathbb{C}$ and by $\sigma(T)$ the spectrum of $T \in \mathcal{L}(X)$.

By applying Lemma 1 and Proposition 1.31 in Reference [12] (or Item (ii) of Proposition 3.2 in Reference [11]), we obtain the following corollary.

Corollary 1. If $T \in \mathcal{L}(X)$ is equicontinuous, then $\sigma(T) \subset S^{1}$.

To complete the proof of Theorem 2, we only need to prove the following lemma. Recall that a linear isometry of a Banach space is a distance preserving linear operator.

Lemma 2. Every linear isometry of a Banach space does not have the shadowing property.

Proof. Let $X$ be a Banach space and $T \in \mathcal{L}(X)$ be a linear isometry. Suppose by contradiction that $T$ has the shadowing property. Fix $\delta>0$ from the equicontinuity of $T$ for $\varepsilon=1$. Take $x \in X$. Choose a sequence $0=q_{0}, q_{1}, \cdots, q_{r}=x$ such that $\left\|q_{i+1}-q_{i}\right\| \leq \delta$ for every $0 \leq i \leq r-1$. Define $\left(p_{i}\right)_{i \in \mathbb{Z}}$ by

$$
p_{i}=\left\{\begin{array}{ccc}
T^{i}\left(q_{0}\right) & \text { if } & i \leq 0 \\
T^{i}\left(q_{i}\right) & \text { if } & 0 \leq i \leq r \\
T^{i}\left(q_{r}\right) & \text { if } & r<i
\end{array}\right.
$$

Since

$$
\left\|T\left(p_{i}\right)-p_{i+1}\right\|=\left\|T^{i+1}\left(q_{i}\right)-T^{i+1}\left(q_{i+1}\right)\right\|=\left\|q_{i+1}-q_{i}\right\| \leq \delta, \quad \forall 0 \leq i \leq r-1,
$$


one has that $\left(p_{i}\right)_{i \in \mathbb{Z}}$ is a $\delta$-pseudo orbit of $T$. Then, there is $z \in X$ such that $\left\|T^{i}(z)-p_{i}\right\| \leq 1$ for every $i \in \mathbb{Z}$. Since $T$ is an isometry, $\left\|z-q_{i}\right\|=\left\|z-T^{i}\left(p_{i}\right)\right\|=\left\|T^{i}(z)-p_{i}\right\| \leq 1$ for every $0 \leq i \leq r$. In particular, $\|z\|=\|z-0\|=\left\|z-q_{0}\right\| \leq 1$ and $\|z-x\|=\left\|z-q_{r}\right\| \leq 1$, so

$$
\|x\| \leq\|z\|+\|z-x\| \leq 2 \text {. }
$$

We conclude that $\|x\| \leq 2$ for every $x \in X$ which is absurd. This completes the proof.

Proof of Theorem 2. Let $X$ be a Banach space and $T \in \mathcal{L}(X)$ be equicontinuous. Define

$$
\|x\|^{\prime}=\sup _{n \in \mathbb{Z}}\left\|T^{n}(x)\right\| .
$$

Clearly, $\|\cdot\|^{\prime}$ is a norm of $X$ and $\|x\| \leq\|x\|^{\prime}$ for every $x \in X$. On the other hand, by Lemma 1 , there is $0<M<\infty$ such that $\left\|T^{n}\right\|<M$ for every $n \in \mathbb{Z}$. It follows that $\left\|T^{n}(x)\right\| \leq\left\|T^{n}\right\| \cdot\|x\| \leq$ $M\|x\|$ for all $n \in \mathbb{Z}$ so $\|x\|^{\prime} \leq M\|x\|$ for every $x \in X$. This proves that $\|\cdot\|$ and $\|\cdot\|^{\prime}$ are equivalent, i.e.,

$$
\|x\| \leq\|x\|^{\prime} \leq M\|x\|, \quad \forall x \in X .
$$

Define $X^{\prime}=X$ equipped with the norm $\|\cdot\|^{\prime}$. By Equation (1), we have that $X^{\prime}$ is a Banach space. Define $T^{\prime}$ as being $T$ in the Banach space $X^{\prime}$. Since

$$
\left\|T^{\prime}(x)\right\|^{\prime}=\sup _{n \in \mathbb{Z}}\left\|T^{n}(T(x))\right\|=\sup _{n \in \mathbb{Z}}\left\|T^{n+1}(x)\right\|=\sup _{n \in \mathbb{Z}}\left\|T^{n}(x)\right\|=\|x\|^{\prime}, \quad \forall x \in X,
$$

we have that $T^{\prime}: X^{\prime} \rightarrow X^{\prime}$ is a linear isometry. In addition, we have that the identity $I: X \rightarrow X^{\prime}$ is a linear homeomorphism by Equation (1), which satisfies $T^{\prime} \circ I=I \circ T$. In particular, $T$ has the shadowing property if and only if $T^{\prime}$ has the shadowing property. But $T^{\prime}$ is an isometry, so it cannot have the shadowing property by Lemma 2 . Then, $T$ cannot have the shadowing property and the proof follows.

Proof of Theorem 3. Let $T \in \mathcal{L}(X)$ be an expansive linear operator with the shadowing property of a Banach space. Suppose by contradiction that $T$ has a nonwandering point $x_{0} \neq 0$. Take $\delta>0$ from the shadowing property for $\epsilon=\frac{\left\|x_{0}\right\|}{4}$. Shrinking $\delta$ if necessary, we can assume $\delta<\frac{\left\|x_{0}\right\|}{2}$. Since $x_{0}$ is nonwandering, there are $x \in X$ and $k>0$ such that $\left\|x-x_{0}\right\|<\frac{\delta}{2}$ and $\left\|T^{k}(x)-x_{0}\right\|<\frac{\delta}{2}$.

Define the sequence $x_{n k+i}=T^{i}(x)$ for $n \in \mathbb{Z}$ and $0 \leq i \leq k-1$. Since $\left\|T^{k}(x)-x\right\|=\| T^{k}(x)-$ $x_{0}\|+\| x-x_{0} \| \leq \frac{\delta}{2}+\frac{\delta}{2}=\delta$, we have that $\left(x_{m}\right)_{m \in \mathbb{Z}}$ is a $\delta$-pseudo orbit. It follows that there is $y \in X$ such that

$$
\left\|T^{n k+i}(y)-x_{n k+i}\right\| \leq \epsilon, \quad \forall n \in \mathbb{Z}, 0 \leq i \leq k-1 .
$$

From this we get

$$
\left\|T^{n k+i}(y)\right\| \leq\left\|T^{n k+i}(y)-x_{n k+i}\right\|+\left\|x_{n k+i}\right\| \leq \epsilon+\sup _{0 \leq i \leq k-1}\left\|T^{i}(x)\right\|,
$$

for every $n \in \mathbb{Z}$ and $0 \leq i \leq k-1$. Since $\sup _{0 \leq i \leq k-1}\left\|T^{i}(x)\right\|<\infty$, the sequence $\left(\left\|T^{m}(y)\right\|\right)_{m \in \mathbb{Z}}$ is bounded. Since $T$ is expansive, $y=0$ by Proposition 19-(c) in Reference [5]. Then, Equation (2) implies $\left\|x_{m}\right\| \leq \epsilon=\frac{\left\|x_{0}\right\|}{4}$ for every $m \in \mathbb{Z}$. By taking $m=0$, we obtain $\|x\| \leq \frac{\left\|x_{0}\right\|}{4}$. Therefore,

$$
\left\|x_{0}\right\| \leq\left\|x-x_{0}\right\|+\|x\|<\frac{\delta}{2}+\frac{\left\|x_{0}\right\|}{4}<\frac{\left\|x_{0}\right\|}{4}+\frac{\left\|x_{0}\right\|}{4}=\frac{\left\|x_{0}\right\|}{2}
$$

which is absurd. This completes the proof.

Author Contributions: Authors contribution was the same for both. 
Acknowledgments: The first author was supported by Chungnam National University, and the second by CNPq-Brazil-303389/2015-0.

Conflicts of Interest: The authors declare no conflicts of interest.

\section{References}

1. Bayart, F.; Matheron, E. Dynamics of Linear Operators; Cambridge Tracts in Mathematics, 179; Cambridge University Press: Cambridge, UK, 2009.

2. Eisenberg, M. Expansive automorphisms of finite-dimensional vector spaces. Fund. Math. 1966, 59, 307-312. [CrossRef]

3. Eisenberg, M.; Hedlund, J.H. Expansive automorphisms of Banach spaces. Pac. J. Math. 1970, 34, $647-656$. [CrossRef]

4. Ombach, J. The shadowing lemma in the linear case. Univ. Iagel. Acta Math. 1994, 31, 69-74.

5. Bernardes, N.C.; Cirilo, P.R.; Darji, U.B.; Messaoudi, A.; Pujals, E.R. Expansivity and shadowing in linear dynamics. J. Math. Anal. Appl. 2018, 461, 796-816. [CrossRef]

6. Morales, C.A. Shadowable points. Dyn. Syst. 2016, 31, 347-356. [CrossRef]

7. Aponte, J.; Villavicencio, H. Shadowable Points for Flows. J. Dyn. Control Syst. 2018, 24, 701-719. [CrossRef]

8. Kawaguchi, N. Properties of shadowable points: Chaos and equicontinuity. Bull. Braz. Math. Soc. 2017, 48, 599-622. [CrossRef]

9. Kawaguchi, N. Quantitative shadowable points. Dyn. Syst. 2017, 32, 504-518. [CrossRef]

10. Utz, W.R. Unstable homeomorphisms. Proc. Am. Math. Soc. 1950, 1, 769-774. [CrossRef]

11. Costakis, G.; Manoussos, A.; Parissis, I. Recurrent Linear Operators. Complex Anal. Oper. Theory 2014, 8, 1601-1643. [CrossRef]

12. Dowson, H.R. Spectral Theory of Linear Operators; London Mathematical Society Monographs, 12; Academic Press, Inc.: Cambridge, MA, USA; Harcourt Brace Jovanovich, Publishers: London, UK; New York, NY, USA, 1978.

(C) 2018 by the authors. Licensee MDPI, Basel, Switzerland. This article is an open access article distributed under the terms and conditions of the Creative Commons Attribution (CC BY) license (http://creativecommons.org/licenses/by/4.0/). 